\footnotetext{
(D) Ligia da Silva Cantanhêde ${ }^{1}$

(iD) Rafaella Lemos Alves²

(iD) Eloise Schott ${ }^{1}$
}

${ }^{1}$ Universidade Federal do Tocantins, Curso de Nutrição. Palmas, Tocantins, Brasil.

2 Universidade de Brasília, Programa de Pós-Graduação em Nutrição Humana. Brasília, Distrito Federal, Brasil.

Correspondência Eloise Schott eloise@uft.edu.br

\section{(In)segurança alimentar e disponibilidade domiciliar de alimentos de moradores da região norte do Estado do Tocantins}

\author{
Food (in)security and home availability of food for the \\ Northern region of the State of Tocantins residents
}

\begin{abstract}
Resumo
Introdução: Estudos que analisam fatores associados à (in)segurança alimentar e nutricional são imprescindíveis para o planejamento de ações e políticas públicas de promoção da saúde. Objetivo: Avaliar a disponibilidade domiciliar de alimentos de acordo com a classificação NOVA e sua associação com a situação de (in)segurança alimentar das famílias. Métodos: Estudo transversal realizado com 199 famílias residentes na microrregião do Bico do Papagaio, no estado do Tocantins. Foram analisados dados socioeconômicos e demográficos das famílias. A situação de insegurança alimentar foi avaliada a partir da Escala Brasileira de Insegurança Alimentar. Para o cálculo da disponibilidade energética diária no domicílio, seguiu-se a metodologia sugerida pela FAO, agrupada conforme o grau de processamento dos alimentos. Utilizou-se o teste qui-quadrado e teste de tendência linear para verificar a associação entre as variáveis. Resultados: No total, 69,9\% da população apresentou algum grau de insegurança alimentar, e 43,2\% não possuía disponibilidade calórica de alimentos suficiente. Observou-se que, conforme aumentava o grau de insegurança alimentar, reduzia-se o valor calórico disponível no domicílio, sendo que a maior contribuição calórica foi advinda dos alimentos in natura e/ou minimamente processados, seguida de ingredientes culinários, alimentos ultraprocessados e de alimentos processados, independentemente do grau de insegurança alimentar $(p<0,05)$. Conclusão: Ressalta-se a importância de avaliar diferentes determinantes relacionados à (in)segurança alimentar nessa região, além de adotar políticas públicas que melhorem as condições de vida das famílias e implementem estratégias que incentivem a produção e consumo de alimentos regionais.
\end{abstract}

Palavras-chave: Alimentação. Disponibilidade de Alimentos. Segurança Alimentar e Nutricional.

\begin{abstract}
Introduction: Studies that review the factors associated with food and nutritional (in)security are essential for the planning of public health promotion actions and policies. Objective: To assess household food availability according to the NOVA classification and its association with the situation of families food (in)security Methods: Cross-sectional study conducted with 199 families living in the Bico do Papagaio microregion, in the State of Tocantins. Families socioeconomic and demographic data were reviewed. The situation of food insecurity was assessed using the Brazilian Food Insecurity Scale. To calculate the daily energy availability at home, the methodology suggested by FAO was followed, grouping according to the degree of food processing. The chi-square test and the linear trend test were used to verify association among
\end{abstract}


the variables. Results: In total, 69.9\% of the population had some degree of food insecurity, and $43.2 \%$ did not have sufficient food calories availability. It was observed that, as the degree of food insecurity increased, the caloric value available in the household got reduced, with the greatest caloric contribution coming from fresh and/or minimally processed foods, followed by culinary ingredients, ultra-processed foods and processed foods, regardless of the level of food insecurity $(p<0.05)$. Conclusion: The importance of evaluating different determinants of food (in)security in this region is emphasized, in addition to the importance of adopting public policies that improve the families' living conditions and help implementing strategies that encourage production and consumption of regional foods.

Keywords: Food. Food Availability. Food and Nutrition Security. 


\section{INTRODUÇÃO}

O acesso à alimentação é um direito de todos, e para garantir uma alimentação adequada e saudável, são necessárias condições básicas de vida, como trabalho, moradia, emprego, educação, saúde e lazer, entre outros. 1,2

No Brasil, esse direito foi firmado por meio da Lei Orgânica de Segurança Alimentar e Nutricional - LOSAN (Lei n 11.346, de 15 de setembro de 2006), que define Segurança Alimentar e Nutricional (SAN) como a realização do direito de todos ao acesso regular e permanente a alimentos de qualidade, em quantidade suficiente, sem comprometer o acesso a outras necessidades essenciais, tendo como base práticas alimentares promotoras de saúde que respeitem a diversidade cultural e que sejam ambiental, cultural, econômica e socialmente sustentáveis. ${ }^{3}$

Para avaliar a condição de (in)segurança alimentar familiar no Brasil, tem sido utilizada a Escala Brasileira de Insegurança Alimentar (EBIA), instrumento que avalia a percepção dos indivíduos em relação ao acesso aos alimentos e sua disponibilidade no domicílio. ${ }^{4}$ A Organização das Nações Unidas para a Alimentação e Agricultura (FAO) utiliza tradicionalmente o indicador de medida de disponibilidade calórica média diária per capita para avaliar e acompanhar o grau de vulnerabilidade à carência alimentar de diferentes países. ${ }^{5}$

A evolução da disponibilidade de alimentos nos domicílios brasileiros, com base nas últimas Pesquisas de Orçamentos Familiares (POFs), indica que os alimentos in natura ou minimamente processados e ingredientes culinários estão perdendo espaço para alimentos processados e ultraprocessados. ${ }^{6}$ Essa transição do padrão alimentar da população tem sido associada ao intenso aumento da prevalência de obesidade, diabetes mellitus e várias outras doenças crônicas relacionadas à alimentação, além de contribuir para o aumento nos índices das deficiências nutricionais.?

Segundo Monteiro et al., em países desenvolvidos, alimentos ultraprocessados já são predominantes na alimentação. Assim, pesquisas voltadas para a obtenção de indicadores de consumo alimentar como as POFs destacam-se por serem uma importante fonte de resultados. ${ }^{9}$

Ressalta-se portanto, a necessidade de realizar o levantamento de determinantes que reflitam desde o acesso ao alimento até as condições desse acesso, fornecendo indicadores úteis e confiáveis para avaliação e monitoramento das condições de insegurança alimentar, uma vez que o desenvolvimento de estudos que analisam fatores associados à (in)segurança alimentar e nutricional são imprescindíveis para o planejamento de ações e políticas públicas de promoção da saúde. 10,11

Além disso, a classificação NOVA, baseada no grau de processamento dos alimentos, tem sido amplamente utilizada em estudos epidemiológicos de consumo alimentar, qualidade da alimentação e condições de saúde dos indivíduos e desenvolvimento de guias alimentares, como o Guia Alimentar para a População Brasileira. ${ }^{12,13}$

Neste sentido, o presente estudo teve como objetivo avaliar a disponibilidade de alimentos e a (in)segurança alimentar de famílias residentes na microrregião do Bico do Papagaio, no norte do Estado do Tocantins.

\section{MÉTODOS}

Trata-se de estudo transversal de base populacional, inserido em um estudo maior intitulado "Prevalência e fatores associados à (in)segurança alimentar e nutricional no Estado do Tocantins", que foi conduzido na área urbana de 22 municípios tocantinenses. Para este trabalho, foram utilizados dados preliminares de quatro municípios pertencentes à microrregião do Bico do Papagaio - Araguatins, Augustinópolis, Praia Norte e Santa Terezinha do Tocantins -, perfazendo 199 domicílios. 
Foi utilizado um plano de amostragem complexa por conglomerados, dividido em três estágios: primeiro, foram sorteadas amostras de municípios com base em uma divisão das microrregiões do Tocantins proporcionalmente ao tamanho populacional; depois foram classificados e sorteados aleatoriamente os setores censitários em cada município; a seguir, um quarteirão, e neste uma esquina que foi o ponto de partida para os domicílios. Entrevistou-se a quantidade de famílias necessária até alcançar o quantitativo estabelecido no cálculo amostral.

Realizou-se treinamento prévio dos entrevistadores e um estudo-piloto; posteriormente, iniciou-se a coleta dos dados no período de setembro de 2016 a julho de 2017. Para a coleta de dados, foram feitas visitas domiciliares e utilizados os seguintes procedimentos: aplicação de questionário semiestruturado ao chefe de família, contendo dados como escolaridade, raça/cor, idade, sexo do entrevistado, total de pessoas residentes no domicílio, classificação por idade destes residentes, renda familiar per capita e participação no programa Bolsa Família do Governo Federal.

A situação de (in)segurança alimentar do domicílio foi avaliada a partir da Escala Brasileira de Insegurança Alimentar (EBIA), uma escala psicométrica que mede diretamente a percepção da (in)segurança alimentar familiar dos últimos três meses. ${ }^{14} \mathrm{~A}$ EBIA foi validada a partir da escala americana e disponibilizada como instrumento de fácil aplicação e baixo custo para diagnóstico da (in)segurança alimentar no Brasil..15

A disponibilidade de alimentos no domicílio foi verificada a partir de questionário de disponibilidade de alimentos aplicado pelo pesquisador ao chefe de família, que informou sobre aquisição de alimentos e bebidas para consumo domiciliar dos últimos 30 dias. O registro incluiu a descrição do alimento/bebida, quantidade adquirida e unidade de medida.

Os alimentos foram agrupados de acordo com a classificação NOVA, baseada na extensão e finalidade do processamento industrial (in natura e minimamente processados, ingredientes processados para culinária, alimentos processados e ultraprocessados) proposta por Monteiro et al., ${ }^{8}$ a fim de avaliar a contribuição de cada grupo de alimentos na disponibilidade alimentar e sua relação com a segurança alimentar do domicílio.

Para o cálculo da disponibilidade energética diária no domicílio, foi seguida a metodologia sugerida pela Organização das Nações Unidas para a Alimentação e Agricultura (FAO) e apresentada por Smith, segundo a qual as famílias com disponibilidade alimentar abaixo de 2.500kcal/per capita/dia foram consideradas em situação de insegurança alimentar. ${ }^{16}$

Os dados foram tabulados em duplicata, a fim de identificar erros de digitação, no programa Microsoft Excel 2010®. A análise estatística foi feita com auxílio do programa Stata versão 14.0. Após realização do teste de normalidade dos dados, as variáveis foram descritas em frequência e mediana, e intervalo interquartil. Utilizou-se o teste qui-quadrado para verificar a relação entre a insegurança alimentar observada pelo método da FAO e pela EBIA. O teste de tendência linear foi aplicado para averiguar a associação entre a disponibilidade de calorias totais e por grupo alimentar per capita, e a situação de (in)segurança alimentar domiciliar.

O projeto de pesquisa foi submetido ao Comitê de Ética em Pesquisa da Universidade Federal de Viçosa (CAAE: 55435716.6.0000.5153), e os participantes assinaram previamente o Termo de Consentimento Livre e Esclarecido, autorizando sua participação.

\section{RESULTADOS}

Em relação ao perfil do público estudado, observa-se, a partir da tabela 1, que há predominância de chefes de família com idade entre 17 e 49 anos (59,3\%); pardos (56,8\%), do sexo feminino (75,4\%) e 49,3\% possuíam menos 
de oito anos de estudo. Em 37,7\% das famílias, a renda per capita foi de até meio salário-mínimo, 47 famílias (23,6\%) recebiam benefício socioassistencial do governo e 50,3\% dos domicílios possuíam mais de três moradores.

Quanto à situação de (in)segurança alimentar, verifica-se, a partir dos resultados da EBIA, que 69,8\% dos domicílios apresentaram algum grau de insegurança alimentar. Já pelo método de disponibilidade de alimentos proposto pela FAO, 43,2\% das famílias encontravam-se nesta condição devido à baixa disponibilidade calórica do domicílio (tabela 1). Além disso, constatou-se que quanto maior o grau de insegurança alimentar determinado pela EBIA, maior era o percentual de famílias com insegurança alimentar detectado pelo método da $F A O(p=0,04)$ (dado não apresentado em tabela).

Tabela 1. Caracterização das famílias estudadas segundo dados socioeconômicos e demográficos. Microrregião do Bico do Papagaio, Tocantins, Brasil, 2016/2017 (n=199).

\begin{tabular}{|c|c|c|}
\hline Variáveis & $\mathrm{N}$ & $\%$ \\
\hline Chefe de família & 199 & 100,0 \\
\hline \multicolumn{3}{|l|}{ Sexo } \\
\hline Feminino & 150 & 75,4 \\
\hline Masculino & 49 & 24,6 \\
\hline \multicolumn{3}{|l|}{ Idade } \\
\hline $17-49$ & 118 & 59,3 \\
\hline 50-99 & 81 & 40,7 \\
\hline \multicolumn{3}{|l|}{ Cor/Raça } \\
\hline Branco & 39 & 19,6 \\
\hline Preto & 44 & 22,1 \\
\hline Pardo & 113 & 56,8 \\
\hline Indígena & 03 & 1,5 \\
\hline \multicolumn{3}{|l|}{ Escolaridade } \\
\hline$<4$ anos & 53 & 26,7 \\
\hline 4-8 anos & 45 & 22,6 \\
\hline$>8$ anos & 101 & 50,7 \\
\hline \multicolumn{3}{|l|}{ Domicílios } \\
\hline \multicolumn{3}{|c|}{ Renda familiar per capita (em salários-mínimos) } \\
\hline Até $1 / 2$ & 75 & 37,7 \\
\hline $1 / 2-1$ & 62 & 31,2 \\
\hline$>1-2$ & 27 & 13,6 \\
\hline$>2$ & 07 & 3,5 \\
\hline Não informado & 28 & 14,0 \\
\hline \multicolumn{3}{|l|}{ Beneficiários do Programa Bolsa Família } \\
\hline Sim & 47 & 23,6 \\
\hline Não & 152 & 76,4 \\
\hline \multicolumn{3}{|l|}{ Número de moradores } \\
\hline De 1 à 3 & 99 & 49,7 \\
\hline De 4 à 6 & 84 & 42,2 \\
\hline$\geq 7$ & 16 & 8,1 \\
\hline \multicolumn{3}{|l|}{ (in)segurança alimentar (EBIA) } \\
\hline Segurança alimentar & 60 & 30,3 \\
\hline Insegurança alimentar leve & 94 & 47,3 \\
\hline Insegurança alimentar moderada/grave & 45 & 22,6 \\
\hline \multicolumn{3}{|l|}{ (in)segurança alimentar (FAO) } \\
\hline Segurança alimentar & 113 & 56,8 \\
\hline Insegurança alimentar & 86 & 43,2 \\
\hline
\end{tabular}


Quanto aos alimentos disponíveis com maior frequência nos domicílios, destacam-se, entre os alimentos in natura ou minimamente processados, arroz, feijão, carne bovina, carne de frango e cebola. Entre os ingredientes culinários, foram mais encontrados açúcar, sal e óleo. Já os alimentos processados mais relatados foram: pão francês, queijo, milho enlatado e sardinha enlatada; e por fim, os alimentos ultraprocessados: margarina, molho pronto de tomate, biscoito salgado, refrigerante e macarrão instantâneo (dados não apresentados em tabela).

A tabela 2 mostra que quanto maior o grau de insegurança, menor é a disponibilidade calórica domiciliar. Os alimentos in natura são os que apresentam maior contribuição calórica para todos os graus de (in)segurança alimentar, seguidas de ingredientes processados, alimentos ultraprocessados e, por último, alimentos processados. Observa-se relação estatisticamente significante entre a (in)segurança alimentar e a disponibilidade calórica per capita total, de alimentos in natura e minimamente processados, alimentos processados e ultraprocessados $(p<0,05)$.

Tabela 2. Disponibilidade calórica per capita total e por grau de processamento dos alimentos e a situação de (in)segurança alimentar. Bico do Papagaio, Tocantins, Brasil, 2016/2017 (n=199).

\begin{tabular}{|c|c|c|c|c|c|}
\hline $\begin{array}{l}\text { (In)segurança } \\
\text { alimentar }\end{array}$ & $\begin{array}{l}\text { Kcal } \\
\text { total } \\
\text { per capita }\end{array}$ & $\begin{array}{l}\text { Kcal } \\
\text { alimentos } \\
\text { in natura e minimamente } \\
\text { processados }^{a}\end{array}$ & $\begin{array}{l}\text { Kcal } \\
\text { ingredientes } \\
\text { culinários }^{a}\end{array}$ & $\begin{array}{l}\text { Kcal } \\
\text { alimentos } \\
\text { processados }^{a}\end{array}$ & $\begin{array}{l}\text { Kcal } \\
\text { alimentos } \\
\text { ultraprocessados }\end{array}$ \\
\hline & $p=0,024$ & $p=0,047$ & $p=0.065$ & $p=0,00$ & $p=0,00$ \\
\hline Segurança alimentar & $\begin{array}{l}2889,56 \\
(2307,77-3938,23)\end{array}$ & $\begin{array}{l}1861,90 \\
(1333,89-2338,98)\end{array}$ & $\begin{array}{l}569,49 \\
(405,76-757,06)\end{array}$ & $\begin{array}{l}107,52 \\
(56,86-198,89)\end{array}$ & $\begin{array}{l}354,41 \\
(221,16-495,16)\end{array}$ \\
\hline $\begin{array}{l}\text { Insegurança alimentar } \\
\text { leve }\end{array}$ & $\begin{array}{l}2582,13 \\
(1862,17-3422,62)\end{array}$ & $\begin{array}{l}1624,68 \\
(1155,71-2317,01)\end{array}$ & $\begin{array}{l}488,68 \\
(323,11-742,73)\end{array}$ & $\begin{array}{l}64,51 \\
(20,91-122,63)\end{array}$ & $\begin{array}{l}239,65 \\
(125,68-407,02)\end{array}$ \\
\hline $\begin{array}{l}\text { Insegurança alimentar } \\
\text { moderada/grave }\end{array}$ & $\begin{array}{l}1437,06 \\
(2433,66-3922,03)\end{array}$ & $\begin{array}{l}1366,02 \\
(982,81-2525,64)\end{array}$ & $\begin{array}{l}435,11 \\
(297,82-765,04)\end{array}$ & $\begin{array}{l}28,03 \\
(7,97-98,10)\end{array}$ & $\begin{array}{l}148,35 \\
(91,72-338,58)\end{array}$ \\
\hline
\end{tabular}

Nota: ${ }^{a}$ Valores de Kcal apresentados em mediana e intervalo interquartil.

\section{DISCUSSÃO}

Nossos resultados apontam que 69,9\% (n=139) da população avaliada por meio da EBIA apresentou algum grau de insegurança alimentar. Diferentes prevalências de insegurança alimentar (IA) nas várias regiões do Brasil são demonstrativas de desigualdades regionais, visto que o Norte e Nordeste apresentam as mais desfavoráveis situações de IA, representando um importante problema de saúde pública.

Dados da PNAD (2013) revelam que 36,1\% dos domicílios da Região Norte e 37,6\% das famílias tocantinenses estavam em IA. ${ }^{14}$ Destaca-se, ainda, que os resultados da POF 2017-2018 apontam que 36,7\% dos domicílios brasileiros avaliados encontravam-se com algum grau de insegurança alimentar, sendo que as regiões Norte e Nordeste continuam apresentando a situação mais desfavorável, com 43,0\% e 49,7\%, respectivamente, o que indica aumento da insegurança alimentar no país, que estava em declínio desde 2004. ${ }^{17}$ Tais prevalências, apesar de expressivas, ainda são menores que a encontrada neste estudo. 
O aumento da prevalência de insegurança alimentar pode ser relacionado, dentre outros, ao perfil de governança, às políticas econômicas de austeridade e às crises econômicas. ${ }^{18}$ Nesse contexto, a Política Nacional de Segurança Alimentar Nutricional tem como desafio a consolidação das políticas públicas nessas regiões, devido a suas desigualdades econômicas e geopolíticas. ${ }^{19}$

Em relação às condições sociais, Braga20 observou que no Brasil há uma relação entre insegurança alimentar e gênero, mostrando maior vulnerabilidade nos domicílios chefiados por mulheres. Da mesma forma, domicílios cuja pessoa de referência era de raça preta ou parda registraram prevalências de insegurança alimentar maiores em todas as suas dimensões do que os domicílios com pessoa de referência branca. ${ }^{14}$ Achados do presente estudo demonstram a vulnerabilidade dos domicílios avaliados, uma vez que, em sua maioria, seus chefes de família eram mulheres e/ou pardos.

Menores renda e escolaridade, ausência de vínculo empregatício e saneamento básico estão relacionados com a insegurança alimentar. ${ }^{21}$ Dentre estes, destaca-se a renda, fator que pode influenciar na escolha dos alimentos e no perfil alimentar. De acordo com Claro, ${ }^{22}$ taxas e tributos aplicados a alguns grupos de alimentos podem afetar o consumo alimentar de determinadas classes sociais, uma vez que isso eleva o valor final dos produtos ao consumidor. A participação de grupos de alimentos compostos por leite e derivados, frutas, verduras e legumes, gordura animal, bebidas alcoólicas e refeições prontas tende a aumentar uniformemente conforme o nível de rendimentos familiares. ${ }^{23}$

No entanto, graus agudos de deficiência alimentar podem ser sinônimo de fome, embora nem sempre a carência alimentar expresse automaticamente tal fenômeno. Em situação de renda desfavorável, o indivíduo troca a qualidade dos alimentos e também da dieta, procurando otimizar seus recursos financeiros. Posteriormente, com pouco ou nenhum recurso financeiro, começa a diminuir a quantidade de alimento disponível para consumo da família, prioritariamente dos adultos. Além de a pobreza se relacionar com a insegurança alimentar, existe ainda uma preocupação atual, em estudos mais recentes, com a relação entre IA e obesidade. ${ }^{24}$

A dieta básica do brasileiro é caracterizada pelo consumo de café, pão, arroz, feijão e carne bovina, além da presença de sucos e refrigerantes e pouca participação de frutas e hortaliças, que se aproximam dos alimentos mais disponíveis nos domicílios estudados. ${ }^{25}$ As mudanças no comportamento alimentar têm se refletido em todas as classes sociais e em todas as regiões do país, indicando que a alimentação dos brasileiros vem se caracterizando pela introdução de alimentos ultraprocessados de alta densidade energética e bebidas com adição de açúcar. ${ }^{25}$

Ressalta-se que o baixo consumo alimentos como frutas, verduras e legumes está relacionado habitualmente à situação de insegurança alimentar. ${ }^{24}$ Apesar disso, a POF 2017/2018 apontou uma participação elevada de alimentos in natura ou minimamente processados e de ingredientes culinários nas regiões Norte e Nordeste, entre famílias com menor renda. ${ }^{6}$

Almeida et al. ${ }^{26}$ observaram, em seu estudo, que dentre os grupos alimentares com ingestão abaixo do mínimo recomendado, destacam-se frutas, hortaliças e leites e derivados. Esses dados refletem um alto percentual de inadequação para diversos nutrientes, como fibras, vitaminas e minerais, o que compromete a segurança alimentar e nutricional dos indivíduos. Embora alguns hábitos tradicionais de alimentação ainda sejam mantidos, destaca-se o declínio no consumo de alimentos básicos, como arroz e feijão, nas áreas metropolitanas do país, ao contrário do que foi encontrado neste estudo, em que os domicílios se localizam em uma região interiorana que ainda preserva hábitos alimentares básicos e regionais no padrão alimentar.

Ressalta-se, ainda, que a disponibilidade de alimentos no domicílio é determinada por vários fatores, tais como renda, condições de acesso a estabelecimentos de comercialização de alimentos, ${ }^{27}$ produção para consumo próprio e produção local de alimentos. ${ }^{28}$ Assim, torna-se importante mensurar a disponibilidade de alimentos para uma melhor avaliação da insegurança alimentar no âmbito domiciliar. ${ }^{29,30}$ 


\section{CONCLUSÃO}

Os domicílios pesquisados apresentaram maior contribuição calórica de alimentos in natura e/ou minimamente processados, independentemente do grau de insegurança alimentar. É importante destacar, no entanto, o elevado percentual de insegurança alimentar encontrado nessa população, bem como sua relação com a redução do valor calórico disponível nos domicílios.

Com isso, observa-se a necessidade de adotar políticas públicas intersetoriais que propiciem a melhoria das condições de vida das famílias, bem como promovam igualdade de gênero e raça/cor.

Ressalta-se, por fim, a importância de avaliar diferentes determinantes relacionados à (in)segurança alimentar na região, para melhor compreender as necessidades dessa população, além da implementação de estratégias que incentivem a produção e o consumo de alimentos regionais e ações de educação alimentar e nutricional que estejam de acordo com a cultura alimentar e a realidade local, buscando garantir, assim, a segurança alimentar e nutricional.

\section{REFERÊNCIAS}

1. De Oliveira Pinheiro AER. Alimentação saudável e a promoção da saúde no contexto da segurança alimentar e nutricional. Revista Saúde em Debate. 2005; 29(70):125-139.

2. Rocha B, Lima A, Almeida PC. Insegurança alimentar relacionada à área de residência em município do Semiárido brasileiro. Cad Saúde Colet. 2014; 22(2):205-11. https://doi.org/10.1590/1414-462X201400020015.

3. Brasil. Lei $n^{\circ}$ 11.346, de 15 de setembro de 2006. Cria o Sistema Nacional de Segurança Alimentar e Nutricional (SISAN) com vistas em assegurar o direito humano à alimentação adequada e dá outras providências. Diário Oficial da União. Brasília, 18 set 2006.

4. Santos LP, Lindemann IL, Motta JVS, Mintem G, Bender E, Gigante DP. Proposta de versão curta da Escala Brasileira de Insegurança Alimentar. Rev Saúde Publ. 2014; 48(5):783-89. https://doi.org/10.1590/S00348910.2014048005195.

5. Food And Agriculture Organization (FAO). Measurement and assessment of food deprivation and undernutrition. International Scientific Symposium, Rome, 26-28 June, 2002. Rome: FAO; 2003. [Acesso em: Jun. 2019]. Disponível em: http://www.fivims.net/EN/ISS.htm.

6. Instituto Brasileiro de Geografia e Estatística (IBGE). Pesquisa de orçamentos familiares 2017-2018: avaliação nutricional da disponibilidade domiciliar de alimentos no Brasil / IBGE, Coordenação de Trabalho e Rendimento. Rio de Janeiro: IBGE, 2020.

7. Levy-Costa RB, Sichieri R, Pontes NS, Monteiro CA. Disponibilidade domiciliar de alimentos no Brasil: distribuição e evolução (1974-2003). Rev. Saúde Publ. 2005; 39(4):530-540. https://doi.org/10.1590/S0034-89102005000400003.

8. Monteiro CA, Cannon G, Levy RB, Moubarac JC, Jaime P, Martins AP, et al. NOVA. The star shines bright. [Food classification.Public health]. World Nutr. 2016; 7(1-3):28-38.

9. Instituto Brasileiro de Geografia e Estatística (IBGE). Pesquisa de Orçamentos Familiares 2008-2009 - POF. Rio de Janeiro, 2010.

10. Salles-Costa R, Pereira RA, Vasconcellos MTL, Veiga GV, Marins VMR, Jardim BC, et al. Associação entre fatores socioeconômicos e insegurança alimentar: estudo de base populacional na Região Metropolitana do Rio de Janeiro, Brasil. Rev. Nutr. 2008.21(supl 0):99-109. 
11. Segall-Corrêa AM. Insegurança alimentar medida a partir da percepção das pessoas. Estud. Avanc. 2007; 21(60):143154.

12. Monteiro CA, Cannon G, Lawrence M, Louzada MLC, Machado PP. Ultra-processed foods, diet quality, and health using the NOVA classification system. Food and Agriculture Organization of the United Nations - FAO. Rome, 2019. [Acesso em: abr 2020] Disponível em: http://www.fao.org/fsnforum/resources/fsn-resources/ultra-processed-foodsdiet-qualityand-health-using-nova classification.

13. Brasil. Ministério da Saúde. Guia alimentar para a população brasileira. 2a Ed. Brasília: Ministério da Saúde; 2014.

14. Instituto Brasileiro de Geografia e Estatística (IBGE). Pesquisa Nacional por Amostra de Domicílios 2013 - Suplemento de Segurança Alimentar. Rio de Janeiro: IBGE, 2014.

15. Ministério do Desenvolvimento Social e Combate à Fome. Secretaria de Avaliação e Gestão da Informação. Estudo Técnico No. 01/2014 Escala Brasileira de Insegurança Alimentar - EBIA: análise psicométrica de uma dimensão da Segurança Alimentar e Nutricional, 2014. [Acesso em: nov 2020]. Disponível em: https://aplicacoes.mds.gov.br/sagirmps/simulacao/estudos_tecnicos/pdf/73.pdf

16. Smith LC. The use of household expenditure surveys for the assessment of food insecurity. In: Proceedings Measurement and assessment of food deprivation and undernutrition. International Scientific Symposium. Roma, 2002. [Acesso em: abr 2019]. Disponível em: http://www.fao.org/3/y4249e/y4249e00.htm

17. Instituto Brasileiro de Geografia e Estatística (IBGE). Pesquisa de orçamentos familiares 2017-2018: análise da segurança alimentar no Brasil / IBGE, Coordenação de Trabalho e Rendimento. - Rio de Janeiro : IBGE, 2020.

18. FAO - Food and Agriculture Organization of the United Nations. The State of Food Security and Nutrition in the World. Building resilience for peace and food security. Rome: FAO, 2017. [Acesso em: nov 2020]. Disponível em: http://www.fao.org/3/a-i7695e.pdf

19. Câmara Interministerial de Segurança Alimentar e Nutricional (CAISAN). Plano Nacional de Segurança Alimentar e Nutricional: 2012/2015. Brasília, DF: CAISAN; 2011.

20. Braga CAS. Insegurança alimentar e nutricional em duas perspectivas: índice de insegurança alimentar dos estados brasileiros e a mulher como chefe em diferentes configurações de divisão do tempo. 2018. Dissertação (Mestrado em Economia Aplicada) - Viçosa, MG.

21. Bezerra TA, Olinda RA, Pedraza DF. Insegurança alimentar no Brasil segundo diferentes cenários sociodemográficos. Cienc. Saúde Colet. 2017; 22(2):637-651. https://doi.org/10.1590/1413-81232017222.19952015

22. Claro RM, Monteiro CA. Renda familiar, preço de alimentos e aquisição domiciliar de frutas e hortaliças no Brasil. Rev. Saúde Publ. 2010; 44(6):1014-1020. https://doi.org/10.1590/S0034-89102010000600005

23. Levy RB, Claro RM, Mondini L, Sicheri R, Monteiro CA. Distribuição regional e socioeconômica da disponibilidade domiciliar de alimentos no Brasil em 2008-2009. Rev. Saúde Publ. 2012; 46(1):06-15. https://doi.org/10.1590/S003489102011005000088

24. Panigassi G, Segal-Correa AM, Marin-León L, Pérez-Escamilla R, Maranha LK, Sampaio MFA et.al. Intra-family Food Insecurity and Profile of Food Consumption. Rev. Nutr. 2008; 21(suppl) 135-144 http://dx.doi.org/10.1590/S141552732008000700012.

25. Souza AM, Pereira RA, Yokoo EM, Levy RB, SICHIERI R. Alimentos mais consumidos no Brasil: Inquérito nacional de alimentação 2008-2009. Rev. Saúde Publ. 2013; 47(supl 1):190s-199s. https://doi.org/10.1590/S003489102013000700005 
26. Almeida JA, Santos AS, Nascimento MAO, Oliveira JVC, Silva DG, Mendes-Netto RS, et al. Fatores associados ao risco de insegurança alimentar e nutricional em famílias de assentamentos rurais. Cienc. Saúde Colet. 2017; 22(2):479488. https://doi.org/10.1590/1413-81232017222.27102015.

27. Sisk C, Sharkey JR, Mclntosh WA, \& Anding J. Using multiple household food inventories to measure food availability in the home over 30 days: a pilot study. Nutr Journ. 2010; 9(19).

28. Harris-Fry H, Azad K, Kuddus A, Shaha S, Nahar B, Hossen M, et al. Socio-economic determinants of household food security and women's dietary

29. diversity in rural Bangladesh: a cross-sectional study. J Health Popul Nutr. 2015 ; 33:2. https://doi.org/10.1186/s41043-015-0022-0.

30. Osório MM, Ribeiro MDA, Costa EC, Silva SPDO, Fernandes CE. Disponibilidade familiar de alimentos na Zona da Mata e Semiárido do Nordeste do Brasil. Rev. Nutr. 2009; 22(3):319-329..

\section{Colaboradores}

Cantanhêde LS participou da análise e interpretação dos dados, redação do artigo e revisão da versão final; Alves RL participou da coleta de dados, revisão e aprovação da versão final; Schott E participou da concepção e desenho do estudo, coleta de dados, revisão e aprovação da versão final.

Conflito de interesses: as autoras declaram não haver conflito de interesses.

Recebido: 18 de maio de 2020

Aceito: 03 de fevereiro de 2021 